\title{
On radical classes of hemirings
}

\author{
Hvedri Inassaridze ${ }^{1}$, Le Hoang $\mathrm{Mai}^{2}$, Nguyen Xuan Tuyen ${ }^{3}$ \\ 1 A. Razmadze Mathematical Institute of Tbilisi State University \\ 2 Dong Thap University, Vietnam \\ 3 College of Pedagogy, Hue University, Vietnam \\ E-mail: inassari@yahoo.com, Ihmai@dthu.edu.vn, nxtuyenvn@yahoo.com
}

\begin{abstract}
Based on the concept of accessible subhemirings and inspired by the work on the general Kurosh-Amitsur radical theory for rings, this paper studies the lower radical classes and the hereditary radical classes of hemirings. We characterize radical classes of hemirings, and construct a lower radical class from a homomorphically closed class. We provide a necessary and sufficient condition under which an upper radical class of hemirings becomes hereditary and prove that an upper radical class of a regular class of semirings is hereditary. Besides, we show that the Brown-McCoy radical class and a Jacobson-type radical class are hereditary.
\end{abstract}

2000 Mathematics Subject Classification. . 16Y60, 8A30, 06A99, 06F99, 18G05, 18 G99.

Keywords. accessible subhemiring, radical class of hemirings, lower radical class, upper radical class, hereditary radical class of hemirings.

\section{Introduction}

A very general setting for Kurosh-Amitsur radical theory was presented in [1] by Márki, Mlitz and Wiegandt, developed in a category which satisfies six axioms of a rather general nature. In a hemiring setting, Olson and co-authors [2-5] started to develop a Kurosh-Amitsur radical theory. Motivated by the Kurosh-Amitsur radical theory for rings (see [6], for example), Hebisch, Weinert and Morak [7-10] developed a Kurosh-Amitsur radical theory for hemirings. Although the main concepts in the radical theory for hemirings are defined similarly to those in the radical theory for rings, detailed developments (e.g., considerations, proofs) for the former may be different and more complicated. In this paper, based on the concept of accessible subhemirings and inspired by the work on the general Kurosh-Amitsur radical theory in [1], we study the lower radical classes and the hereditary radical classes of hemirings.

The paper is organized as follows. We describe in Section 2 some important notions and facts on hemirings and semimodules, as well as the Kurosh-Amitsur radical theory of hemirings. More details on the Kurosh-Amitsur radical theory of hemirings can be found in [8-11], and on hemiring theory in [12]. In Section 3, we characterize radical classes of hemirings (Proposition 3.1), construct a radical class from a homomorphically closed class (Theorem 3.2) and prove that it is a lower radical class (Corollary 3.4). In Section 4, we give a necessary and sufficient condition under which an upper radical class of hemirings becomes hereditary (Theorem 4.1) and prove that the upper radical class of a regular class of semirings is hereditary (Theorem 4.2). In addition, we note that the Brown-McCoy radical class and the radical class $\mathbb{J}_{s}$ are hereditary (Corollaries 4.3 and 4.6). 


\section{Preliminaries}

Recall from [12] that a hemiring $R$ is an algebra $(R,+, \cdot, 0)$ such that the following conditions are satisfied:

(1) $(R,+, 0)$ is a commutative monoid with identity element 0 ;

(2) $(R, \cdot)$ is a semigroup;

(3) multiplication distributes over addition on either side;

(4) $0 r=0=r 0$ for all $r \in R$.

A hemiring $R$ is called a semiring if its multiplicative semigroup $(R, \cdot)$ is a monoid with identity element 1. The notions of (two-sided) ideal, left ideal and right ideal of a hemiring $R$ are defined similarly as for rings. The $k$-closure $\bar{I}=\{r \in R \mid r+i \in I$ for some $i \in I\}$ of an ideal $I$ is an ideal of $R$. An ideal $I$ of a hemiring $R$ is called a $k$-ideal (or subtractive) if $\bar{I}=I$; that is, for all $x, a \in R$, if $x+a, a \in I$ then $x \in I$. Denote by $\mathcal{I}(R)$ and $\mathcal{K}(R)$ the sets of all ideals and all $k$-ideals of $R$, respectively.

As usual, a left $R$-semimodule is a commutative monoid $\left(M,+, 0_{M}\right)$ together with a scalar multiplication $(r, m) \mapsto r m$ from $R \times M$ to $M$ that satisfies the following identities for all $r, r^{\prime} \in R$ and $m, m^{\prime} \in M$ :

(1) $\left(r r^{\prime}\right) m=r\left(r^{\prime} m\right)$;

(2) $r\left(m+m^{\prime}\right)=r m+r m^{\prime}$;

(3) $\left(r+r^{\prime}\right) m=r m+r^{\prime} m$;

(4) $r 0_{M}=0_{M}=0 \mathrm{~m}$.

Right $R$-semimodules are defined dually. For semirings, we require semimodules to be unitary in the usual sense. Denote by $\mathcal{M}_{R}$ and ${ }_{R} \mathcal{M}$ the categories of all right and left $R$-semimodules, respectively. For a left $R$-semimodule $M$, the ideal $(0: M)_{R}=\{r \in R \mid r M=0\}$ of $R$ is called the annihilator of $M$.

Any ideal $I$ of a hemiring $R$ induces on $R$ a congruence relation $\equiv_{I}$, which is referred to as Bourne relation [12, p.78] and is given by: $r \equiv_{I} r^{\prime}$ iff there exist elements $i_{1}, i_{2} \in I$ such that $r+i_{1}=r^{\prime}+i_{2}$. Denote the factor hemiring $R / \equiv_{I}$ by $R / I$. It is easy to see that $\equiv_{I}$ and $\equiv_{\bar{I}}$ on $R$ coincide for any ideal $I$ of $R$, and hence $R / I=R / \bar{I}$ holds for all ideals $I$ of $R$.

Katsov and Nam in [11] briefly reviewed the Kurosh-Amitsur radical theory of the category $\mathbb{H}$ of all hemirings, which was developed by various scientists in $[2-5,7-10]$. In this theory, a nonempty subclass $\mathbb{U}$ of $\mathbb{H}$ is said to be hereditary if $R \in \mathbb{U}$ implies $\mathcal{I}(R) \subseteq \mathbb{U}$, and homomorphically closed if $R \in \mathbb{U}$ implies $\varphi(R) \in \mathbb{U}$ for each homomorphism $\varphi$ of $R$. If $\mathbb{U}$ is both hereditary and homomorphically closed, then it is said to be universal. Similarly to the radical theory of rings, there are three equivalent approaches to the Kurosh-Amitsur radical theory of hemirings, by means of radical classes, of radical operators, and of semisimple classes. These approaches are independently defined in a fixed universal class $\mathbb{U} \subseteq \mathbb{H}$ of hemirings. Define $\mathbb{T}=\{S \in \mathbb{H}|| S \mid=1\}$ as the class of all trivial hemirings. A class $\mathbb{M}$ is said to be regular if for every $R \in \mathbb{M}$ and every nonzero ideal $I$ of $R$, there exists a nonzero surjective homomorphism $I \rightarrow J$ such that $J \in \mathbb{M}$.

A nonempty subclass $\mathbb{R}$ in a fixed universal class $\mathbb{U} \subseteq \mathbb{H}$ is called a radical class of $\mathbb{U}$ if $\mathbb{R}$ satisfies the following two conditions [8]:

(1) $\mathbb{R}$ is homomorphically closed;

(2) for every hemiring $R \in \mathbb{U} \backslash \mathbb{R}$, there is a $k$-ideal $K \in \mathcal{K}(R) \backslash\{R\}$ such that $\mathcal{I}(R / K) \cap \mathbb{R}=\{0\}$.

A mapping $\rho: \mathbb{U} \rightarrow \mathbb{U}$ is called a radical operator in $\mathbb{U}$ if it assigns to each hemiring $R \in \mathbb{U}$ a $k$-ideal $\rho(R) \in \mathcal{K}(R) \subseteq \mathbb{U}$ such that the following conditions are satisfied for all $S, T \in \mathbb{U}[8]$ : 
(1) $\varphi(\rho(S)) \subseteq \rho(\varphi(S))$ for each homomorphism $\varphi: S \rightarrow T$;

(2) $\rho(S / \rho(S))=\{0\}$

(3) if $\rho(T)=T$ is an ideal of $S$ then $T \subseteq \rho(S)$;

(4) $\rho(\rho(S))=\rho(S)$.

\section{On lower radical classes}

As in the case of rings (see, for example, [6]), a subhemiring $S$ of a hemiring $R$ is said to be accessible if there exists a finite sequence of subhemirings $S_{1}, \ldots, S_{n}$ of $R$ such that $S=S_{1} \leq S_{2} \leq \ldots \leq S_{n}=$ $R$, where $S_{i}$ is an ideal of $S_{i+1}$. The category of hemirings considered in this paper satisfies the six axioms in [1]. The relation " $S$ is an accessible subhemiring of a hemiring $R$ " is an " $M$-relation" in the sense of [1], and it is homomorphically closed and transitive. Therefore, based on the general Kurosh-Amitsur radical theory developed in [1], radical classes of hemirings can be characterized in terms of accessible subhemirings, like in the ring case (see [6, Theorem 3.1.9]), as follows.

Proposition 3.1. For a subclass $\mathbb{R}$ in a universal class $\mathbb{U} \subseteq \mathbb{H}$, the following conditions are equivalent:

(1) $\mathbb{R}$ is a radical class of $\mathbb{U}$;

(2) $\mathbb{R}$ satisfies the following two properties:

(2a) If $R \in \mathbb{R}$, then for every nonzero surjective homomorphism $R \rightarrow S$ there exists a nonzero accessible subhemiring $I$ of $S$ such that $I \in \mathbb{R}$;

(2b) If $R \in \mathbb{U}$ and for every nonzero surjective homomorphism $R \rightarrow S$ there exists a nonzero accessible subhemiring $I$ of $S$ such that $I \in \mathbb{R}$, then $R \in \mathbb{R}$.

Let $\mathbb{A}$ be an arbitrary class of hemirings. In view of the characterization of radical classes in [8, Theorem 4.7], the intersection of any set of radical classes containing $\mathbb{A}$ is itself a radical class containing $\mathbb{A}$, and this radical class is called the lower radical class of $\mathbb{A}$ and denoted by $\mathfrak{L} \mathbb{A}$. For rings there are several constructions of the lower radical. In [14], Zulfiqar carried over the construction of Watters [16] to hemirings. Now we do the same with the lower radical construction of Lee [15] (cf. [6, Theorem 3.3.2 and Proposision 3.3.3]).

Theorem 3.2. If $\mathbb{A}$ is a homomorphically closed subclass in a universal class $\mathbb{U} \subseteq \mathbb{H}$ then the subclass $Y \mathbb{A}$, defined as the class of all $R \in \mathbb{U}$ such that every nonzero homomorphic image of $R$ has a nonzero accessible subhemiring in $\mathbb{A}$, is a radical class of $\mathbb{U}$ that contains $\mathbb{A}$.

Corollary 3.3. If $\mathbb{R}$ is a radical class in a universal class $\mathbb{U} \subseteq \mathbb{H}$, then $Y \mathbb{R}=\mathbb{R}$.

Corollary 3.4. If $\mathbb{A}$ is a homomorphically closed class in universal class $\mathbb{U} \subseteq \mathbb{H}$, then $Y \mathbb{A}$ is the lower radical class of $\mathbb{A}$.

The proof of Theorem 3.2 is similar to that of Theorem 3.3.2 in [6]. Corollary 3.3 follows immediately from Theorems 3.2 and 3.7 in [10], and Theorem 7.1 in [8]. Corollary 3.4 is an immediate consequence of Corollary 3.3.

\section{On hereditary radical classes}

Let $\mathbb{M}$ be a regular class in a universal class $\mathbb{U} \subseteq \mathbb{H}$. The class $\mathcal{U} \mathbb{M}$, defined as the class of all $R \in \mathbb{U}$ such that any nonzero homomorphic image $S$ of $R$ does not belong to $\mathbb{M}$, is a radical class of $\mathbb{U}$. Moreover, each radical class $\mathbb{R}$ in $\mathbb{U}$ with the property $\mathbb{M} \cap \mathbb{R}=\mathbb{T}$ satisfies $\mathbb{R} \subseteq \mathcal{U} \mathbb{M}$ (Theorem 
5.3 in [8]). The radical class $\mathcal{U} \mathbb{M}$ is called the upper radical class of $\mathbb{M}$. Next, we give results for hereditary radical classes of hemirings, by generalizing those for rings (Theorems 3.2.14 and 3.2.16 in $[6])$.

Theorem 4.1. Let $\mathbb{M}$ be a regular class in a universal class $\mathbb{U} \subseteq \mathbb{H}$. Then the upper radical class $\mathcal{U} \mathbb{M}$ is hereditary if and only if $\mathbb{M}$ satisfies the following condition: if $I$ is a nonzero ideal of $S$ and $A \in \mathbb{M}$ is a nonzero homomorphic image of $I$, then there exists a nonzero homomorphic image $B$ of $S$ such that $B \in \mathbb{M}$.

Proof. Suppose that $\mathcal{U} \mathbb{M}$ is hereditary, $I$ is a nonzero ideal of $S$ and $A \in \mathbb{M}$ is a nonzero homomorphic image of $I$. By definition of $\mathcal{U} \mathbb{M}$, we have $I \notin \mathcal{U} \mathbb{M}$, and hence $S \notin \mathcal{U} \mathbb{M}$ because $\mathcal{U} \mathbb{M}$ is hereditary. Thus, $S$ has a nonzero homomorphic image $B \in \mathbb{M}$. Conversely, suppose that $\mathbb{M}$ satisfies the above condition and $I$ is a nonzero ideal of $S \in \mathcal{U M}$. If there exists a nonzero $A \in \mathbb{M}$ such that it is a homomorphic image of $I$ then, according to the above condition, $S$ has a nonzero homomorphic image $B \in \mathbb{M}$ (contradiction). Thus, $I$ does not have a nonzero homomorphic image in $\mathbb{M}$; that is, $I \in \mathcal{U} \mathbb{M}$.

Q.E.D.

Next we prove that the upper radical class of a regular class of semirings is hereditary.

Theorem 4.2. If $\mathbb{M}$ is a regular class of semirings in a universal class $\mathbb{U} \subseteq \mathbb{H}$, then the upper radical class $\mathcal{U} \mathbb{M}$ is hereditary.

Proof. Suppose that $S \in \mathcal{U} \mathbb{M}$ has an ideal $I$ and a nonzero surjective homomorphism $\varphi: I \rightarrow A$ with $A \in \mathbb{M}$. Then $I / \operatorname{Ker} \varphi \cong A$, where $\operatorname{Ker} \varphi=\left\{(x, y) \in I^{2} \mid \varphi(x)=\varphi(y)\right\}$. Since $A \in \mathbb{M}$, we have $I / \operatorname{Ker} \varphi \in \mathbb{M}$. On the other hand, $I / K \operatorname{Ker} \varphi$ is a semiring with identity element $\bar{e}$ for some $e \in I$. By [11, Lemma 3.14], the relation $\varrho$ on $S$, defined by $\forall a, b \in S$, a $\varrho b \Leftrightarrow(e a e, e b e) \in \operatorname{Ker} \varphi$, is a congruence on $S$ and the natural map $\pi: S / \varrho \rightarrow I / \operatorname{Ker} \varphi$ given by $\bar{r} \mapsto \overline{\text { ere }}$ is a semiring isomorphism. This shows that $I / \operatorname{Ker} \varphi \in \mathbb{M}$ is a nonzero homomorphic image of $S$. Therefore, by Theorem $4.1, \mathcal{U} \mathbb{R}$ is hereditary.

Q.E.D.

Following El Bashir et al. [13], a semiring $R$ is said to be ideal-simple if 0 and $R$ are its only ideals. Consider the class $\mathbb{S}$ of all ideal-simple semirings in $\mathbb{H}$. It is obvious that $\mathbb{S}$ is a regular class of semirings in $\mathbb{H}$, and hence, the class $\mathcal{U} \mathbb{S}=\{R \in \mathbb{H} \mid R$ has no nonzero homomorphic images in $\mathbb{S}\}$ is a radical class, called the Brown-McCoy radical class, in $\mathbb{H}$. This radical class has been considered among others by Katsov and Nam [11]. Theorem 4.2 yields now the following property, which is well known for rings.

Corollary 4.3. The Brown-McCoy radical class $\mathcal{U} \mathbb{S}$ is hereditary.

A left $R$-semimodule $M$ is said to be simple if $R M \neq\{0\}$ and there are only trivial subsemimodules of, as well as congruences on, $M$. It was proved in [11] that the mapping $J_{s}: \mathbb{H} \rightarrow \mathbb{H}$, defined by $J_{s}(R)=\cap\left\{(0: M)_{R} \mid M \in \sum_{R}\right\}$ where $\sum_{R}=\left\{{ }_{R} M \in{ }_{R} \mathcal{M} \mid M\right.$ is simple $\}$, is a radical operator in $\mathbb{H}$. Hence, by [9, Theorem 2.6], the set $\mathbb{J}_{s}=\left\{R \in \mathbb{H} \mid J_{s}(R)=R\right\}$ is a radical class of $\mathbb{H}$. We will next show that $\mathbb{J}_{s}$ is hereditary. First, we need the following useful lemma, whose proof is contained in the proof of [11, Proposition 3.5(iii)], although the statement of this lemma is not formulated there explicitly. 
Lemma 4.4. Let $I$ be an ideal in a hemiring $R$. We have:

(1) if $M$ is a simple left $R$-semimodule, then either $I M=\{0\}$ or $M$ is a simple left $I$-semimodule;

(2) a simple left $I$-semimodule $M$ can be extended to a simple left $R$-semimodule $M$, by the scalar multiplication $r\left(\sum a_{i} m_{i}\right)=\sum\left(r a_{i}\right) m_{i}$ for all $a_{i} \in I, m_{i} \in M$ and $r \in R$.

Theorem 4.5. For an ideal $I$ of a hemiring $R, J_{s}(I)=I \cap J_{s}(R)$.

Proof. Because $J_{s}$ is a radical operator in $\mathbb{H}$, we have $J_{s}(I) \subseteq I \cap J_{s}(R)$, according to [8, Theorem 6.2]. We will now show that $I \cap J_{s}(R) \subseteq J_{s}(I)$. Indeed, assume that $x \in I \cap J_{s}(R)$ and $x \notin(0: M)_{I}$ for some simple left $I$-semimodule $M$. Then ${ }_{I} M$ may be extended to a simple left $R$-semimodule ${ }_{R} M$, according to Lemma 4.4. Therefore, $x \notin(0: M)_{R}$; that is, $x \notin J_{s}(R)$. This contradicts the assumption that $x \in I \cap J_{s}(R)$. Hence, $x \in(0: M)_{I}$ for all simple left $I$-semimodules $M$; that is, $x \in J_{s}(I)$.

Q.E.D.

From Theorem 4.5 above and Theorem 6.3 in [8], we have the following corollary.

Corollary 4.6. The radical class $\mathbb{J}_{s}$ is hereditary.

\section{References}

[1] L. Márki, R. Mlitz and R. Wiegandt. A general Kurosh-Amitsur radical theory. Commun. Algebra, 16:249-305, 1988.

[2] D. M. Olson and T. L. Jenkins. Radical theory for hemirings. J. Nature. Sci. Math., 23:23-32, 1983.

[3] D. M. Olson and A. C. Nance. A note on radical for hemirings. Quaestiones Mathematicae, 12:307-314, 1989.

[4] D. M. Olson, G. A. P. Heyman and L. H. LeRoux. Weakly special classes of hemirings. Quaestiones Mathematicae, 15:119-126, 1992.

[5] D. M. Olson, L. H. LeRoux and G. A. P. Heyman. Three special classes for hemirings. Quaestiones Mathematicae, 17:205-215, 1994.

[6] J. Gardner and R. Wiegandt. Radical Theory of Rings. CRC, 2003.

[7] U. Hebisch and H. J. Weinert. Radical theory for semirings. Quaestiones Mathematicae, 20(4):647-661, 1997.

[8] B. Morak. On the radical theory for semirings. Contributions to Algebra and Geometry, 40(2):533-549, 1999.

[9] U. Hebisch and H. J. Weinert. On the interrelation between radical theories for semirings and rings. Commun. Algebra, 29:109-129, 2001.

[10] U. Hebisch and H. J. Weinert. Semisimple classes of semirings. Algebra Colloquium, 2:177-196, 2002 . 
[11] Y. Katsov and T. G. Nam. On radicals of semirings and related problems. Commun. Algebra, 42:5065-5099, 2014.

[12] J. Golan. Semirings and their Applications. Kluwer Academic Publishers, Dordrecht-BostonLondon, 1999.

[13] R. El Bashir, J. Hurt, A. Jančařík and T. Kepka. Simple commutative semirings. J. Algebra, 236:277-306, 2001.

[14] M. Zulfiqar. A note on lower radicals of hemirings. Bulletin of the Korean Mathematical Society, 45(4):757-762, 2008.

[15] Y.-L. Lee. On the construction of lower radical properties. Pacific J. Math., 28:393-395, 1969.

[16] J. F. Watters. Lower radicals in associative rings. Canad. J. Math., 21:466-476, 1969. 